\title{
180. Some Observations on the Cortico-thalamic Fiber Connections in the Monkey
}

\author{
By Shizu SAKaI \\ Department of Neuroanatomy,*) Institute of Brain Research, \\ Faculty of Medicine, University of Tokyo \\ (Comm. by Teizo Ogawa, M.J.A., Oct. 12, 1967)
}

Earlier studies failed to reveal the detailed organization of the cortico-thalamic projections, although the existence have been widely established..$^{2), 7), 10,11)}$ In late decade, the organizations of cortical projections to the subcortical nuclei, especially to the thalamic nuclei, have been studied in our laboratory, using cat materials..$^{1,4), 5)}$ These results prompted the present investigation on the cortico-thalamic projections in monkeys.

Materials and methods. Sixteen adult monkeys (Macaca fuscata and Macaca mulatta) were used in this study. Lesions were made either bilaterally (in 14 monkeys) or unilaterally (in 2 monkeys) in various parts of the frontal and pericentral gyri and the superior parietal lobulus. Two or three weeks later, the animals were killed by an overdose of Nembutal and perfused via the heart with $3.000 \mathrm{ml}$ of $10 \%$ neutral formalin. The Nauta-Gygax method was employed on serial frozen sections of every tenth cut frontally or sagittally at $30 \mu$. Each adjoining sections were stained with the Nissl or spielmeyer method in order to identify the thalamic nuclei on the basis of the atlas of Olszewski. ${ }^{13)}$

Results and discussion. 1) Projections to the ventral thalamic nuclei (Fig. 1).

Projections of the pericentral cortical areas to the ventral thalamic nuclei were ipsilateral. The precentral area was divided, for the sake of descriptions, into three parts, a, b, and $c$ as shown in Fig. 4. Ablations of ' $a$ ' was followed by abundant preterminal degenerating fibers in the nucleus ventralis lateralis, pars oralis (VLo) and by some in the anterior part of the nucleus ventralis lateralis, pars caudalis (VLc) (Fig. 1). On the other hand, following lesions in ' $b$ ', considerably many preterminal degenerating fibers were demonstrated in the rostral part of the nucleus ventralis posterior lateralis, pars oralis (VPLo), in the dorsal region of the caudal part of VPLo and in the posterior part of VLc, while the ventral region of the caudal part of VPLo showed only few, if any,

*) Director: Prof. T. Kusama. 


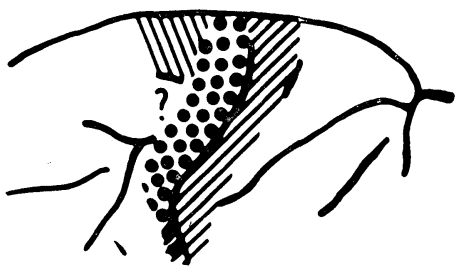

Fig. 1

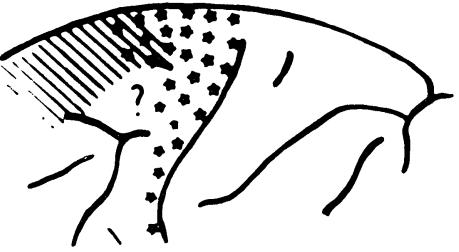

Fig. 3

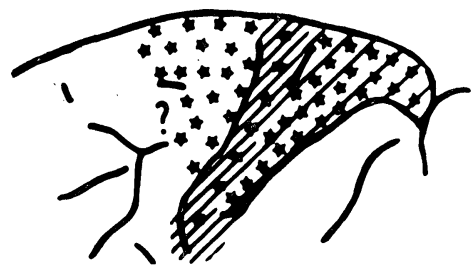

F i g. 2

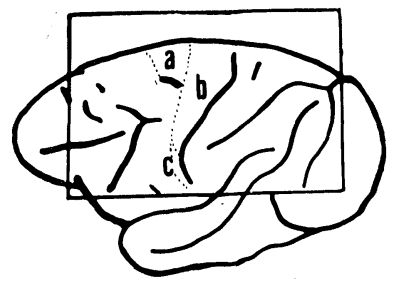

F i g. 4

Diagrammatic representation of the differential cortical origin of projections to the thalamus.

Fig. 1. Projections to VLo $(\backslash \backslash \backslash)$ originated primarily from the anterior part of the precentral area, namely ' $a$ ' in Fig. 4, while those to VPLo and VLc $(\therefore)$ arose from the rest of this gyrus, namely ' $b$ ' and ' $c$ '. Projections to VPLc and VPM $(/ / /)$ occured primarily from the postcentral gyrus.

Fig. 2. Projections to LP $\left(*_{*}^{*}\right)$ arose from the presentral and postcentral gyri and the superior parietal lobulus. Projections to the Pul.o $(/ / /)$ orginated from the postcentral gyrus and the superior parietal lobulus.

Fig. 3. Projections to $\operatorname{Pf}(\backslash \backslash \backslash)$ primarily originated from the premotor area, while those to the CnMd $(* *)$ arose from the precentral area.

Fig. 4. Diagrammatic representation of the lateral surface of the hemisphere in the monkey. The square indicated the part drawn in Fig. 1,2, and 3.

*) The question marks in Fig. 1,2, and 3 mean to indicate that projections of this area remain unsolved and to need further investigation.

preterminal degenerating fibers.

Following lesions in the postcentral gyrus, preterminal degenerating fibers were observed in the nucleus ventralis posterior lateralis, pars caudalis (VPLc) and in the nucleus ventralis posterior medialis (VPM). In these projections a topographical arrangement was indicated; after small lesions placed in its medial third, preterminal degenerating fibers were confined in the lateral part of VPLc, while lesions in the lateral third were followed by preterminal fibers degenerations in VPM. According to previous findings in cats in our laboratory, ${ }^{4), 51}$ 
cortical projections to VP were organized in a somatotopical fashion.

In monkeys, moreover, projections from the precentral gyrus to VPLo and VLc showed a similar topographical organization: the more medial part of the precentral gyrus projected to more lateral part of VPLo, and vice versa. Therefore, the most lateral part of this gyrus, namely ' $c$ ' in Fig. 4, sent fibers to the most medial parts of VPLo and of VLc. This cortical area, however, projected also to VPM and the dorsolateral extremity of VPMpc, suggesting that this cortical area may be both motor and somatic sensory (Fig. 1).

In our cases no noticeable preterminal degenerating fibers were found in the following ventral nuclei: the nucleus ventralis anterior (VA), the nucleus ventralis lateralis, pars medialis $\left(\mathrm{VL}_{m}\right)$, the area $\times$ and the nucleus ventralis posterior inferior (VPI).

2) Projections to the lateral thalamic nuclei (Fig. 2).

The pericentral gyri and the superior parietal lobulus projected ipsilaterally to the lateral thalamic nuclei. The superior parietal lobulus, the precentral and postcentral gyri sent massive, moderate and small amount of fibers to the nucleus lateralis posterior (LP), respectively.

On the other hand, the postcentral gyrus gave rise to more numerous fibers to the nucleus pulvinaris, pars oralis (Pul.o) than the superior parietal lobulus did.

In contrast with the postcentral projections to the ventral nuclei, which showed a somatotopical organization as mentioned above, its projections to Pul.o were observed to terminate almostly in the same region of Pul.o in spite of different sites of postcentral lesions.

According to previous findings in our laboratory in cat materials, ${ }^{5)}$ the most prominent projection area to LP was localized in the middle suprasylvian gyrus and the anterior two-thirds of the lateral gyrus. The projections were also observed to occur from the posterior sigmoid gyrus and the posterior part of the coronal gyrus, though much less in amount. However, any projection was scarcely found to arise from the anterior sigmoid gyrus and the anterior part of the coronal gyrus ${ }^{4}$ : These cortical areas were regarded as the motor cortex in cats. ${ }^{4), 5)}$ In monkeys, on the contrary, projection area to LP extended to the precentral gyrus (Fig. 2).

3) Projections to the intralaminar thalamic nuclei (Fig. 3).

Projection fibers from the precentral area and the rostrally adjoining region terminated in the different parts of the intralaminar thalamic nuclei, with overlapping.

Following lesions in the precentral area, many of preterminal degenerating fibers were found in the nucleus centrum medianum 
$(\mathrm{CnMd})$ and in the ventral part of the nucleus centralis lateralis (CL). On the other hand, lesions in the rostrally adjoining cortex produced preterminal fiber degenerations in the nucleus parafascicularis (Pf), the dorsal part of CL and the lateral part of the nucleus medialis dorsalis (MD). Recently, Petras ${ }^{14), 15)}$ and others ${ }^{6), 9)}$ reported on the cortico-thalamic projections to CnMd with the topographical organization in the monkey and chimpanzee.

Acknowledgements. The author wishes to express her appreciation to Prof. T. Kusama for his courtesy and invaluable advice. The author is also grateful to Dr. T. Ogawa for his encouragement and criticism, and to Mrs. K. Toyoshima and Miss T. Takahashi for their technical assistance.

\section{References}

1) Akiba, H.: Projections from the sensory motor cortex to the subcortical nuclei superior to the mesencephalic level in cats. J. Chiba Med. Soc., 36, 688-709 (1960) (in Japanese).

2) Auer, J.: Terminal degeneration in the diencephalon after ablation of frontal cortex in the cat. J. Anat., 90, 30-41 (1956).

3) Crouch, R. L.: The nuclear configuration of the thalamus of macaca rhesus. J. Comp. Neurol., 59, 451-483 (1934).

4) Kawana, E., and Kusama, T.: Projection of the sensory motor cortex to the thalamus, the dorsal column nucleus, the trigeminal nucleus and the spinal cord in the cat. Folia Psychiat. Neurol. Japonica, 18, 337-380 (1964).

5) Kusama, T., et al.: Projections of the motor, somatic sensory, auditory and visual cortices in cats. Progress in Brain Research, vol. 21A, ed. by T. Tokizane and J. P. Schade, Elsevier pub. comp. (1966).

6) Kuypers, H. G. J., and Lawrance D. G.: Cortical projections to the red nucleus and the brain stem in the rhesus monkey, Brain Research, 4, 151-188 (1967).

7) Levin, P. M.: The efferent fibers of the frontal lobe of the monkey. Macaca Mulatta. J. Comp. Neurol., 63, 369-419 (1936).

8) Mabuchi, M., and Kusama, T.: The cortico-rubral projection in the Cat. Brain Research, 2, 254-273 (1966).

9) Mehler, W. R.: Futher Notes on the Centre Median Nucleus of Luys. The thalamus, ed. by D. P. Purpura and M. D. Yahr, Columbia Univ. Press., 109-127 (1966).

10) Mettler, F. A.: Corticofugal fiber connections of the cortex of Macaca Mulatta. The frontal region. J. Comp. Neurol., 61, 509-542 (1935).

11) - Corticofugal fiber connections of the cortex of Macaca Mulata, The parietal region. J. Comp. Neurol., 62, 263-291 (1935).

12) Nauta, W. J. H., and Gygax, P. A.: Silver Impregnation of Degenerating Axons in the Central Nervous System: a modified technique. Stain Tech., 29, 9193 (1954).

13) Olszewski, J.: The Thalamus of the Macaca Mulatta. Basal, S. Karger (1952).

14) Petras, J. M.: Some Fiber Connections of the Precentral and Postcentral Cortex with the Basal Ganglia, Thalamus, and Subthalamus. Trans. Amer. Neurol. Asso. C, 274-275 (1965a). 
15) - Fiber degeneration in the basal ganglia and diencephalon following lesions in the precentral and postcentral cortex of the monkey (Macaca Mulatta; with addition observations in the chimpanzee). 8th Int. Anat. Cong. Wiesbaden, Germany (1965b).

16) Walker, A. E.: The Primate Thalamus. Univ. Chicago Press. Chicago (1938). 